\title{
Modeling Multi-Plant Capacitated Lot Sizing Problem with Interplant Transfer
}

\author{
Amitkumar Patil \\ Department of Mechanical Engineering, \\ Malaviya National Institute of Technology Jaipur, Rajasthan, India. \\ E-mail: 2019rme9109@mnit.ac.in, Orcid ID: 0000-0003-4972-2533. \\ Gaurav Kumar Badhotiya \\ Department of Mechanical Engineering, \\ Graphic Era (Deemed to be University), Dehradun, Uttarakhand, India. \\ Corresponding author: gkb.choudhary@gmail.com, Orcid ID: 0000-0001-6680-1001.

\section{Bimal Nepal} \\ Department of Engineering Technology \& Industrial Distribution, \\ Texas A\&M University, College Station, TX 77843, USA. \\ E-mail: nepal@tamu.edu, Orcid ID: 0000-0001-9288-8964. \\ Gunjan Soni \\ Department of Mechanical Engineering, \\ Malaviya National Institute of Technology Jaipur, Rajasthan, India. \\ E-mail: gsoni.mech@mnit.ac.in
}

(Received on January 21, 2021; Accepted on May 7, 2021)

\begin{abstract}
Lot sizing models involve operational and tactical decisions. These decisions may entail multi-level production processes such as assembly operations with multiple plants and limited capacities. Lot sizing problems are widely recognized as NP-hard problems therefore difficult to solve, especially the ones with multiple plants and capacity constraints. The level of complexity rises to an even higher level when there is an interplant transfer between the plants. This paper presents a Genetic Algorithm (GA) based solution methodology applied to large scale multi-plant capacitated lot sizing problem with interplant transfer (MPCLSP-IT). Although the GA has been a very effective and widely accepted meta-heuristic approach used to solve large scale complex problems, it has not been employed for MPCLSP-IT problem. This paper solves the MPCLSP-IT problem in large scale instances by using a genetic algorithm, and in doing so successfully obtains a better solution in terms of computation time when compared to the results obtained by the other methods such as Lagrangian relaxation, greedy randomized adaptive search procedure (GRASP) heuristics, and GRASP-path relinking techniques used in extant literature.
\end{abstract}

Keywords- Multi-plant capacitated lot sizing problem, Inter-plant transfer, Metaheuristics, Genetic algorithm, Production planning.

\section{Introduction}

Due to the globalization of the manufacturing industry, many companies run their production and distribution operations in multiple locations to meet the customer demands on time while minimizing the overall production time and cost (Vercellis, 1999). These objectives can be achieved through effective production planning and by serving customers from multiple plants. To select optimal locations for manufacturing plants costs associated with processing, setup holding and transportation are to be considered (Guinet, 2001). Aytug et al. (2003) noted that in 
International Journal of Mathematical, Engineering and Management Sciences

Vol. 6, No. 3, 961-974, 2021

https://doi.org/10.33889/IJMEMS.2021.6.3.057

production planning and control literature these problems are classified as lot sizing problems. To that end, lot sizing problems are an integral part of the production planning operations that determine the quantity of items that are to be processed and when they are to be processed (Karimi et al., 2003). According to Gruson et al. (2019), lot sizing problems are one of the widely studied problems in the production management literature thanks to their broad applications in production and supply chain planning. Over the years, various extensions to lot sizing problems have been developed to minimize processing costs, holding costs, and transportation costs for a production system. On the other hand, manufacturing companies are faced with challenges in meeting irregular customer demands which are often difficult to forecast. Few examples could be domestic appliances, mattress industry, and food industries as mentioned by Nascimento et al. (2010). Guinet (2001) pointed out that one way to achieve these objectives is by locating a manufacturing facility close to the customers and also with the help of multiple production units. However, our research reveals that only a few studies such as (Carvalho and Nascimento, 2016, 2018; Nascimento et al., 2010; Nascimento and Toledo, 2008; Sambasivan and Schmidt, 2002; Sambasivan and Yahya, 2005) are based on the large scale MPCLSP.

Lot sizing problems are difficult to solve because of a variety of reasons including capacity restrictions, multi-plant, multi-item, and inter-plant transfer (Deleplanque et al., 2013). In the search for the best solutions to complex real-life scenarios within the production planning environment, advances in mathematical optimization techniques and computational software abilities have been utilized (Baccouche, 2005; Hetreux et al., 1996). In Maes et al. (1991), it is mentioned that single item lot sizing problem models belong to complex NP-complete group problems. Interested readers can refer to a detailed review of the capacitated lot sizing problem (CLSP) and its various models in Karimi et al. (2003). Authors mentioned that CLSP problems are NP-hard and due to complexity in solving these problems a large share of the literature associated with the lot sizing problem is based on a single plant capacitated production environment with single or multiple products and multiple time periods.

As noted in Karimi et al. (2003) CLSP with single plant is an NP-hard problem which also makes multi-plant CLSP problem an NP-hard problem. The NP-hard class of problems requires a very high computation time to reach optimal solutions with the help of exact solution approaches. To tackle this problem, researchers have developed heuristics, and metaheuristics approaches that can be used to obtain near-optimal solutions within less computation time (González-Neira and Montoya-Torres, 2017). One such metaheuristic technique is GA, which has been quite popular due to its flexible nature and its effectiveness in solving large scale complex problems (Aytug et al., 2003; Baccouche et al., 2005; Goren et al., 2010; Goren et al., 2012; Jans and Degraeve, 2007). Moreover, compared to other popular metaheuristics, such as PSO and Ant Colony Optimization, GA has better exploration capabilities in the global search space. For instance, A study (Goren et al., 2012) used GA with "fix and optimize" heuristic to solve CLSP with setup carry-over problem. Their GA-based solution was better in quality (for hybrid as well as for pure GA) than that by Tabu search heuristic. Aytug et al. (2003) presented a review of articles published during 1996 and 2002 in which the authors compared the solutions of production and operations management problems that were solved through GA and other methods. Baccouche et al. (2005) employed a hybrid approach by combining the pros of GA and Tabu Search algorithms for solving a generalized assignment problem. The author validated their results by comparing them with branch-and-cut integer programming CPLEX solver for benchmark job-factory assignments problem. Jans and Degraeve (2007) analyzed generic components of metaheuristics (i.e. GA, simulated annealing and Tabu search) and their implementation on dynamic lot sizing 
International Journal of Mathematical, Engineering and Management Sciences

Vol. 6, No. 3, 961-974, 2021

https://doi.org/10.33889/IJMEMS.2021.6.3.057

problems. Likewsie, Goren et al. (2010) summarized critical features and specifications of GA commonly employed to solve various lot sizing problems. The authors conclude that when employed for lot sizing problems, GA outperforms exact solution approaches as well as other heuristics in most of the cases.

Our review of the extant production and operations management literature reveals that, due to the combinatorial nature of the MPCSLP problems, an exact solution-based approach not only takes longer computational time (compared to heuristics) but also often fails to obtain an optimal solution. The level of complexity rises to an even higher level when there is an interplant transfer of parts between the plants. The objective of this paper is to narrow this gap by employing a genetic algorithm based meta-heuristic approach to an MPCSLP-IT problem to obtain an optimal (or near optimal solution) while reducing the overall computation time. With respect to MPCSLPIT problem, this paper addresses three issues surrounding production planning decisions: 1) which machine will be used in production? 2) what part will a machine produce? And, 3) when will the part be produced? Furthermore, the paper also presents multiple experiments on common benchmark instances, initially investigated by Sambasivan and Yahya (2005) and used by other prior researchers to compare solution methodologies for similar problems. Apart from these benchmark instances, this study also includes an additional set of eight large size instances to demonstrate the efficacy of the proposed GA-based framework. Lastly, solution quality and computational time are the two key metrics used in comparing the solutions obtained by the proposed GA based approach and those using other methods available in the literature.

The remainder of the paper is organized as follows. Section 2 presents an overview of related literature. In section 3, we present the formulation of the proposed the MPCLSP-IT problem. Section 4 provides details about the solution technique proposed in this paper. In section 5, the results obtained from numerical experiments are analyzed and compared. Lastly, section 6 concludes the paper by summarizing the key findings of this study along with few directions for future work.

\section{Overview of Related Literature}

The CLSP has been studied extensively in the past few years. Karimi et al. (2003) showed that a multi-item CLSP is an NP-hard problem. Heuristics and metaheuristics such as genetic algorithms, simulated annealing, ant colony optimization, tabu search, etc. have been popular and known to be effective in solving hard combinatorial problems. Jans and Degraeve (2007) reviewed various metaheuristic and heuristic approaches such as dynamic programming, cutting planes, and Lagrangian relaxations that are employed to solve dynamic lot sizing problems that exist in various fields of production planning. The authors imply that metaheuristics produce better results for complex and large problems but are limited because they are problem specific and do not provide lower bounds as in the cases of the other approaches mentioned above. Brahimi et al. (2017) have studied previous research on the dynamic lot sizing problem and observed that most studies are focused on single item problems only.

The CLSP has many extensions, of which the following are studied extensively and observed in real-life situations: set up carryover, setup times and costs, backordering, sequencing, parallel machines, lead times, etc. Interested readers can refer to Quadt and Kuhn (2008), in which the authors have studied a large variety of CLSPs and classified them based on the previously mentioned extensions. Lot sizing models can also be differentiated based on number of plants, number of products, the planning horizon, and if they are single or multi-level. Jans and Degraeve 
International Journal of Mathematical, Engineering and Management Sciences

Vol. 6, No. 3, 961-974, 2021

https://doi.org/10.33889/IJMEMS.2021.6.3.057

(2007) and Buschkühl et al. (2010) have found mathematical programming and genetic algorithm techniques to be comparatively more popular than other solution approaches for multi-level CLSP problems.

There are several practical multi-level CLSP extensions available in the literature (such as Almeder, 2010; Ghirardi and Amerio, 2019; Gopalakrishnan et al., 2001; Hwang and Kang, 2020; Özdamar and Barbarosoglu, 2000; Sahling et al., 2009; Vincent et al., 2020). Likewise, Özdamar and Barbarosoglu (2000) proposed an integrated Lagrangian-simulated annealing approach for multi-level CLSPs with setup times and a backordering extension model. The authors observed that the proposed integrated approach outperforms both the simulated annealing (SA) approach and the hybrid of simulated annealing and genetic algorithm approach. The multi-level CLSP (ML-CLSP) with setup carryover that was solved using tabu search heuristics by Gopalakrishnan et al. (2001) observed a significant total cost reduction. Sahling et al. (2009) obtained high quality results with moderate efforts and optimized a heuristic for the ML-CLSP with multi-period setup carryover and lead time. Almeder (2010) proposed creating a hybrid approach by incorporating the ant algorithm with the Mixed Integer Programming (MIP) solver to solve small or medium sized ML-CLSPs. Ghirardi and Amerio (2019) considered backordering, setup carryovers, and non-identical parallel machines to solve the CLSP. The authors solved this complex problem with a metaheuristic that was based on a feasibility pump algorithm. Vincent et al. (2020) proposed a population-based metaheuristic method to solve the CLSP that contained multiple items, unrelated parallel machines, and setup times. Hwang and Kang (2020) employed a forward dynamic program with Zangwill partitions to solve the two-level lot sizing problem. The authors used a case that was observed in a third-party logistics company which was focused on integrated replenishment and dispatch planning.

Another extension of the lot sizing problem is the combination of the lot sizing and scheduling problems in single model, which can be observed in various industries, such as the glass industry, pipeline industry, etc. Gupta and Magnusson (2005) proposed an ISI (initialize, sequence, improve) heuristic to solve the capacitated lot sizing and scheduling problem. The authors have considered a single machine system with sequence dependent setup times and costs and allowed setup carryover for multiple time periods. Almada-Lobo et al. (2007) studied a single machine, multiple products capacitated lot sizing problem with sequence dependent setup carryover, setup times and costs in a glass industry, and mixed integer linear programming formulations for the same problem.

Brandimarte (2006) and de Armas and Laguna (2019) have also focused on the stochastic demand incorporated in CLSPs. Brandimarte (2006) developed plant location based model formulations that proposed a fix and relax strategy-based heuristic algorithm. de Armas and Laguna (2019) proposed a heuristic based on a combination of mathematical programming and post-processing sequencing to solve the parallel machine system with lot sizing and scheduling for the pipeline industry.

Single plant multi-item CSLPs are NP-hard, along with multi-plant variants of the problem (Karimi et al., 2003). Many heuristics and hybrid heuristics have been developed to solve this type of problem. Sambasivan and Schmidt (2002) developed an MPCLSP model for a large steel corporation in the United States of America. The authors proposed a heuristic method that integrated the routines of lot shifting and lot splitting to solve the problem. Later, Sambasivan and Yahya (2005) investigated the efficiency of a heuristic based on the Lagrangian relaxation of 
capacity constraints in the same model used by Sambasivan and Schmidt (2002) on the case study of a steel corporation in the United States of America. Using the same mathematical formulation, Nascimento et al. (2010) produced better results by employing a GRASP heuristic embedded with a path-relinking strategy. A Lagrangian heuristic was proposed by Deleplanque et al. (2012) to solve MPCLSPs with transfer and shortage capacity models by decomposing it into two subproblems: the master facility location problem and the multi-commodity flow problem. Carvalho and Nascimento (2016) developed a Lagrangian based heuristic using a path relinking strategy that produced even better results for the standard instances of the MPCLSP problem presented in Nascimento and Toledo (2008) and Sambasivan and Yahya (2005). A recent study has employed GA to solve a similar kind of multi-plant capacitated lot sizing problem, however, considered a small size problem with only two plants (Mohammadi et al., 2020).

These studies suggest that relatively few research scholars have tried to develop better solutions to the MPCLSP model with the interplant transfer problem. From this literature, we can observe that a few heuristics have been developed for medium to large sized problems, but no one has examined the effectiveness of any of the metaheuristic approaches. This paper has tried to fill this gap in the research by employing a GA to solve the discussed problem. The following section will present the mathematical model followed in this study.

\section{Mathematical Model}

This study focuses on a single level multi-plant two-echelon capacitated lot sizing model. The description of the problem is as follows: All plants can produce all types of final products on a single machine system. The manufacturing supply chain involves multiple plants and multiple customers. The customer demand is assumed to be known. The production of each product is independent of the production of the preceding and subsequent items. Customer demands are directly satisfied by the corresponding nearest plants. As this model considers interplant transfer, customers only pay the transfer cost of ordering from the nearest plant. If the nearest plant cannot produce enough to fulfill demand, then other plants transfer the required quantity of products. The interplant transfer costs have to be borne by the manufacturer. An inventory of all of the products is allowed and the setup time and setup cost of a product in each particular plant is taken into consideration in this model. Demand, inventory cost, setup cost, and production cost vary with time-period and plants for individual products. Production time and setup time are constant irrespective of plants and products. The following Figure 1 illustrates the interplant transfer studied in this paper.

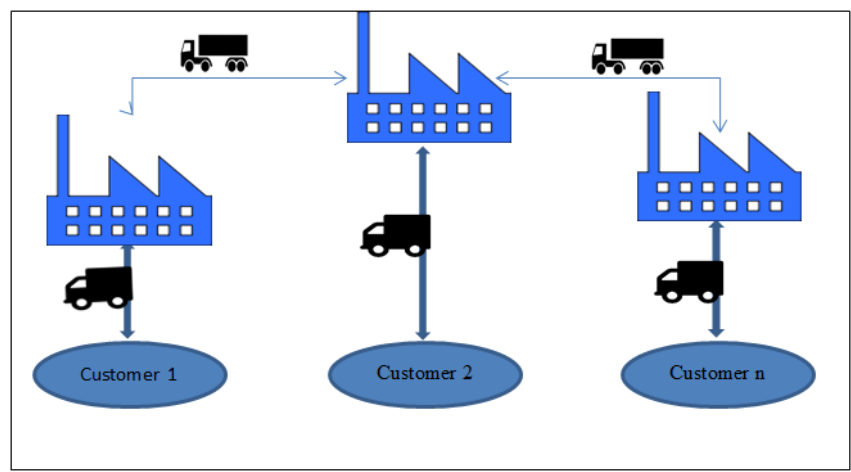

Figure 1. Illustration of multi-customer multi-plant transfer problem. 
International Journal of Mathematical, Engineering and Management Sciences

Vol. 6, No. 3, 961-974, 2021

https://doi.org/10.33889/IJMEMS.2021.6.3.057

Decision variables and parameters:

$\boldsymbol{x}_{i j t}=$ quantity of product $i$ produced at plant $j$ in period $t$;

$\boldsymbol{I}_{i j t}=$ inventory of product $i$ at plant $j$ in period $t$;

$\mathbf{w}_{i j k t}=$ quantity of product $i$ being transported from plant $j$ to plant $k$ in period $t$;

$\mathbf{y}_{i j t}=1$ if a plant $j$ is producing for product $i$ in period $t$

$=0$ else;

$\boldsymbol{D}_{i j t}=$ Demand of product $i$ at plant $j$ in period $t$;

$\boldsymbol{I n v} \boldsymbol{C}_{i j t}=$ Inventory cost of product $i$ at plant $j$ in period $t$;

$S \boldsymbol{C}_{i j t} \quad=$ Setup cost of product $i$ at plant $j$ in period $t$;

$\boldsymbol{P r C}_{i j t}=$ Process cost of product $i$ at plant $j$ in period $t$;

$\boldsymbol{T C}_{i j t} \quad=$ Transportation cost of product $i$ at plant $j$ in period $t$;

$\boldsymbol{P}_{j t} \quad=$ Production capacity of plant $\mathrm{j}$ during period $\mathrm{t}$ in time units;

$\boldsymbol{S T}_{i j t} \quad=$ Setup time for product $i$ at plant $j$ in period $t$;

$\boldsymbol{P r} \boldsymbol{T}_{i j t}=$ Process time for product $i$ at plant $j$ in period $t$;

\section{Objective function:}

Minimize

$\sum_{i} \sum_{j} \sum_{t}\left\{\boldsymbol{P r} \boldsymbol{C}_{\boldsymbol{i j t}} * \mathbf{x}_{i j t}+\boldsymbol{I n v} C_{i j t} * \boldsymbol{I}_{i j t}+\boldsymbol{S} \boldsymbol{C}_{\boldsymbol{i j t}} * \boldsymbol{y}_{i j t}+\left(\sum_{k \neq j} \mathbf{T C}_{\boldsymbol{i j t}} * \boldsymbol{w}_{i j k t}\right)\right\}$

Subject to:

1. Inventory balance constraints:

$$
\boldsymbol{I}_{i j t}=\boldsymbol{I}_{i j t}-1+\mathbf{x}_{i j t}-\sum_{j \neq k} \boldsymbol{w}_{i j k t}+\sum_{l \neq j} \boldsymbol{w}_{i l j t}-\boldsymbol{D}_{i j t} \quad \forall i, j, t .
$$

2. Setup forcing constraints:

$$
\mathbf{x}_{i j t} \leq(100,000) * \boldsymbol{y}_{i j t} \quad \forall i, j, t
$$

3. Production capacity constraints:

$$
\sum_{i}\left(\boldsymbol{P r} \boldsymbol{T}_{i j t} * \mathbf{x}_{i j t}+\boldsymbol{S} \boldsymbol{T}_{i j t} * \boldsymbol{y}_{i j t}\right) \leq \mathbf{P}_{\mathbf{j t}} \quad \forall i, j, t .
$$

4. Non-negativity constraints:

$$
\begin{array}{lll} 
& \mathbf{x}_{\mathrm{ijt}}, \mathrm{I}_{\mathrm{ijt}} \geq 0 & \forall i, j, t \\
& \mathbf{w}_{i j k t} \geq 0 & \forall i, j, t \\
& \mathbf{y}_{i j t} \in\{0,1\} & \forall i, j, t \\
\text { 5. } & \mathrm{I}_{i j 0}=0 & \forall i, j
\end{array}
$$

In this case, the objective function is to minimize the total cost, which includes production, transfer, holding, and setup costs. The first term in the objective function represents the total production cost, the second term represents the total holding cost, the third term represents the total setup cost, and the final term represents the total transfer cost. A plant is said to be setup when a machine starts processing any product in that plant. Before initiating the production of items the machines are setup, which consumes time and cost and is called setup time and setup cost respectively. Setup cost is positive only when a plant is producing any product; otherwise, it is zero. The transfer cost $\mathbf{T} \mathbf{C}_{j k t}$ represents the optimal transfer cost from plant $j$ to plant $k$ in time 
International Journal of Mathematical, Engineering and Management Sciences

Vol. 6, No. 3, 961-974, 2021

https://doi.org/10.33889/IJMEMS.2021.6.3.057

period $t$. Here transfer costs are assumed to be Euclidean costs so that generality is maintained, i.e. $\boldsymbol{w}_{j l t}+\boldsymbol{w}_{l k t} \geq \boldsymbol{w}_{j k t}$.

The constraints 1-5 need to be satisfied to obtain an optimal solution. Constraint 1 is the inventory balance equation. It ensures that the quantity of item $i$ at plant $j$ in period $t$ is produced such that the inventory of item $i$ at plant $j$ from the previous period, the number of item $i$ in period $t$ transferred from plant $j$ and into plant $j$ from other plants satisfies the demand of item $i$ at plant $j$ in period $t$ and the inventory of item $i$ in period $t$ at plant $j$. Constraint 2 verifies that if quantity $i$ is produced at plant $j$ in period $t$, i.e. $\boldsymbol{x}_{i j t}>0$, then the binary variable $\boldsymbol{y}_{i j t}=1$, otherwise 0 . It denotes that plant $j$ is set up for item $i$ in period $t$. Constraint 3 ensures that the available plant capacity is not violated. Constraint 4 imposes the non-negativity of variables $\boldsymbol{x}, \boldsymbol{I}, \boldsymbol{w}$ and ensures that $y$ is a binary variable. Constraint 5 confirms that the first period inventory for all items at all plants is null.

\section{Solution Methodology}

In this paper, genetic algorithm (GA) was utilized to solve a large scale MPCLSP-IT problem. The GA was first introduced by Goldberg and Holland (1988), and since then it has been employed in a wide variety of optimization problems and has remained active to this day Almeder (2010). The GA has proved to be effective when large scale problems need to be solved and there is very little information to process. Due to its flexibility and simplicity, it has been applied to many difficult combinatorial problems (Goldberg and Holland, 1988; Kelly Jr and Davis, 1991). In this paper, efforts are directed towards evaluating its efficiency and effectiveness at solving the MPCLSP-IT problem. GA algorithm is implemented as shown in Figure 2.

1. Create random initial population

2. Evaluate fitness function value

3. Parent chromosome selection

4. Crossover \& Mutation operations

5. Repair Operation

6. New Generation generated

7. Fit population propagated

8. Check termination condition

9. If 8 is true go to 10 or else go to 2

10. Stop

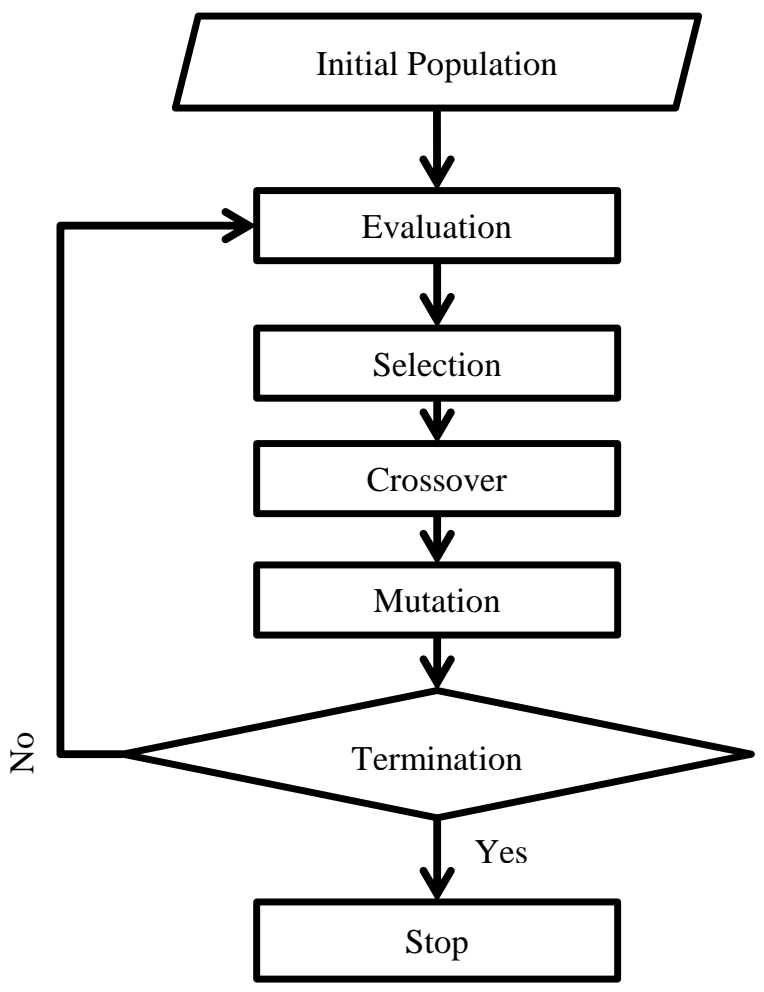

Figure 2. Basic steps of the GA algorithm. 
The genetic algorithm is based on the evolution and survival of the fittest among the population. The basic steps to implement a GA are illustrated in Figure 2, which were adapted from Goren et al. (2012). The first population of candidate solutions was generated with a completely random method. Each member of the population is then converted into chromosomes of search space and has its fitness checked. After the crossover and mutation operations, new chromosomes (child chromosomes) are generated and their fitness and constraint eligibility are evaluated. A new population is created from the fittest of the previous parent chromosomes and recent child chromosomes combined. This new population becomes the parent population and a new child population is created through crossover and mutation operations. This cycle continues until the termination condition is fulfilled.

\section{Numerical Experiments}

To justify the effectiveness of the proposed solution approach, GA is employed to benchmark instances of the MPCLSP from Sambasivan and Yahya (2005) in the MATLAB 2015b version on the PC AMD A10 processor. We also examined even larger scale problems with the product sizes $(2,3)$, plant sizes $(10 \& 15)$, and time periods $(5 \& 10)$. The problem size is in $\mathrm{N}^{*} \mathrm{M}^{*} \mathrm{~T}$ format where, $\mathrm{N}=$ no. of products, $\mathrm{M}=$ no. of plants, and $\mathrm{T}=$ time periods considered. The GA parameters, a crossover probability $\left(\mathrm{p}_{\mathrm{c}}\right)$ of 0.85 , and one bit flip mutation operator are applied. For comparison purposes, all the considered problem cases are solved with IBM ILOG CPLEX software.

\subsection{Problem Parameters}

The parameters mentioned in Table 1 are randomly generated and follow uniform distribution (UD). These are benchmark instances used by Sambasivan and Yahya (2005) as shown in Table 1.

Table 1. Problem parameters.

\begin{tabular}{|c|c|c|}
\hline S. No. & Parameter & Values \\
\hline 1 & Plants & 10,15 \\
\hline 2 & Products & 2,3 \\
\hline 3 & Periods & 5,10 \\
\hline 4 & Setup cost & 100 \\
\hline 5 & Inventory cost & Uniform[10-30] \\
\hline 6 & Production cost & Uniform[20-40] \\
\hline 7 & Transfer cost & Uniform[10-20] \\
\hline 8 & Demand (Random) & 2 \\
\hline 9 & Setup time & $290]$ \\
\hline
\end{tabular}

\subsection{Results and Discussions}

To benchmark the performance of the proposed GA approach in comparison to the MPCLSP method available in the existing literature (e.g. Sambasivan and Yahya, 2005), four instances were run as shown in Table 2. These instances represent 3 products, 3 and 6 machines, and over 5 and 10 time periods. 
International Journal of Mathematical, Engineering and Management Sciences

Vol. 6, No. 3, 961-974, 2021

https://doi.org/10.33889/IJMEMS.2021.6.3.057

Table 2. Computational results for benchmark instances.

\begin{tabular}{|c|c|c|c|c|c|}
\hline Problem size & No. Variables & No. Constraints & Solution gap (\%) & \multicolumn{2}{|c|}{ Computation time (Seconds) } \\
\cline { 4 - 5 } & & & & GA & CPLEX \\
\hline $3 * 3 * 5$ & 225 & 495 & 15.27 & 0.46 & 0.39 \\
\hline $3 * 6 * 5$ & 720 & 3420 & 9.04 & 0.86 & 1.36 \\
\hline $3 * 3 * 10$ & 450 & 990 & 27.15 & 0.81 & 0.42 \\
\hline $3 * 6 * 10$ & 1440 & 6840 & 16.75 & 1.57 & 1.91 \\
\hline
\end{tabular}

Also, a few additional experiments were run by increasing the problem size as described below. These problems included 2 and 3 products, 10 and 15 plants, and over 5 and 10 time periods. The problem size notations are in $\mathrm{N}^{*} \mathrm{M} * \mathrm{~T}$ form, where, $\mathrm{N}=$ no. of products, $\mathrm{M}=$ no. of plants, and $\mathrm{T}=$ no. of time periods. In Table 3, studied instances with solution gaps are presented and compared to the computational times of both the CPLEX and GA solutions.

$$
\text { Solution gap }=\frac{\text { Total cost of CPLEX solution }- \text { Total cost of GA solution }}{\text { Total cost of CPLEX solution }} * 100 \text {. }
$$

The total cost of the CPLEX solution is the objective function value of the solution obtained using IBM ILOG CPLEX; the total cost of the GA solution is the objective function value of the solution obtained through a GA.

Table 3. Computational results for larger problem sizes.

\begin{tabular}{|c|c|c|c|c|c|c|}
\hline \multirow{2}{*}{\multicolumn{2}{|c|}{ Problem size }} & \multirow{2}{*}{ No. Variables } & \multirow{2}{*}{ No. Constraints } & \multirow{2}{*}{ Solution gap (\%) } & \multicolumn{2}{|c|}{ Computation Time (Seconds) } \\
\hline & & & & & GA & CPLEX \\
\hline 1. & $2 * 10 * 5$ & 1200 & 10300 & 7.72 & 1.03 & 4.5 \\
\hline 2. & $2 * 10 * 10$ & 2400 & 20400 & 11.98 & 1.9 & 5.57 \\
\hline 3. & $2 * 15 * 5$ & 2550 & 34050 & 6.60 & 1.89 & 6.46 \\
\hline 4. & $2 * 15 * 10$ & 5100 & 68100 & 10.76 & 2.9 & 8.59 \\
\hline 5. & $3 * 10 * 5$ & 1800 & 15300 & 9.32 & 1.33 & 5.14 \\
\hline 6. & $3 * 10 * 10$ & 3600 & 30600 & 10.21 & 2.51 & 6.9 \\
\hline 7. & $3 * 15 * 5$ & 3825 & 51075 & 9.31 & 2.04 & 5.84 \\
\hline 8. & $3 * 15 * 10$ & 7650 & 102150 & 10.84 & 3.94 & 13.67 \\
\hline
\end{tabular}

The results in Table 2 and Table 3 show that a GA performs better when solving large scale MPCLSP-IT problems. It is observed in Figure 4 that in terms of computation time, a GA outperforms CPLEX in solving large scale CLSPs. All of the solution gaps are within a reasonable range, lying up to a maximum of $12 \%$ (See Table 3). The comparison of CPLEX and GA results in terms of total cost are illustrated in Figure 3. The results obtained in previous studies on the MPCLSP-IT that have used the same benchmark instances as this study are compared and illustrated in Table 4. In Table 4 it can be observed that in terms of a solution gap, the GRASP-path relinking method provides the best solution, but it consumes a larger amount of computation time. The solutions obtained by the GA are not very accurate in terms of a solution gap, but at the cost of a slightly larger solution gap, an optimal solution can be generated in a significantly smaller amount of computation time. For a medium to large scale MPCLSP-IT problem, the GA is a state-of-the-art solution approach that can be used to obtain only slightly less accurate results within an extremely low computation time. 
International Journal of Mathematical, Engineering and Management Sciences

Vol. 6, No. 3, 961-974, 2021

https://doi.org/10.33889/IJMEMS.2021.6.3.057

Table 4. Comparative results table with previous studies.

\begin{tabular}{|c|c|c|c|c|c|c|c|c|c|c|c|}
\hline \multirow{2}{*}{$\begin{array}{l}\text { Sr. } \\
\text { No. }\end{array}$} & \multirow[t]{2}{*}{$\begin{array}{l}\text { Problem } \\
\text { size }\end{array}$} & \multicolumn{2}{|c|}{$\begin{array}{c}\text { Lagrangian } \\
\text { Relaxation }\end{array}$} & \multicolumn{2}{|c|}{ GRASP heuristic } & \multicolumn{2}{|c|}{$\begin{array}{c}\text { GRASP-Path } \\
\text { Relinking } \\
\end{array}$} & \multicolumn{2}{|c|}{$\begin{array}{c}\text { Lagrangean based } \\
\text { shifting heuristic }\end{array}$} & \multicolumn{2}{|c|}{ GA } \\
\hline & & Sol.Gap & Seconds & Sol.Gap & Seconds & Sol.Gap & Seconds & Sol.Gap & Seconds & Sol.Gap & Seconds \\
\hline 1 & $3 * 3 * 5$ & 7.1 & 7.7 & 8.1 & 0.9 & 6.5 & 1.9 & 6.7 & 185 & 15.27 & 0.46 \\
\hline 2 & $3 * 6 * 5$ & 8.2 & 13.6 & 9.1 & 4.8 & 7.1 & 8.4 & 8.4 & 2349 & 9.04 & 0.86 \\
\hline 3 & $3 * 3 * 10$ & 12.1 & 13.5 & 6.5 & 2.2 & 5.1 & 5.7 & 11.5 & 912 & 27.15 & 0.81 \\
\hline 4 & $3 * 6 * 10$ & 9.2 & 11.8 & 10.8 & 14.2 & 7.7 & 35.1 & 9.4 & 4218 & 16.75 & 1.57 \\
\hline & & 9.15 & 11.65 & 8.63 & 5.53 & 6.6 & 12.78 & 9 & 1916 & 13.69 & 0.925 \\
\hline
\end{tabular}

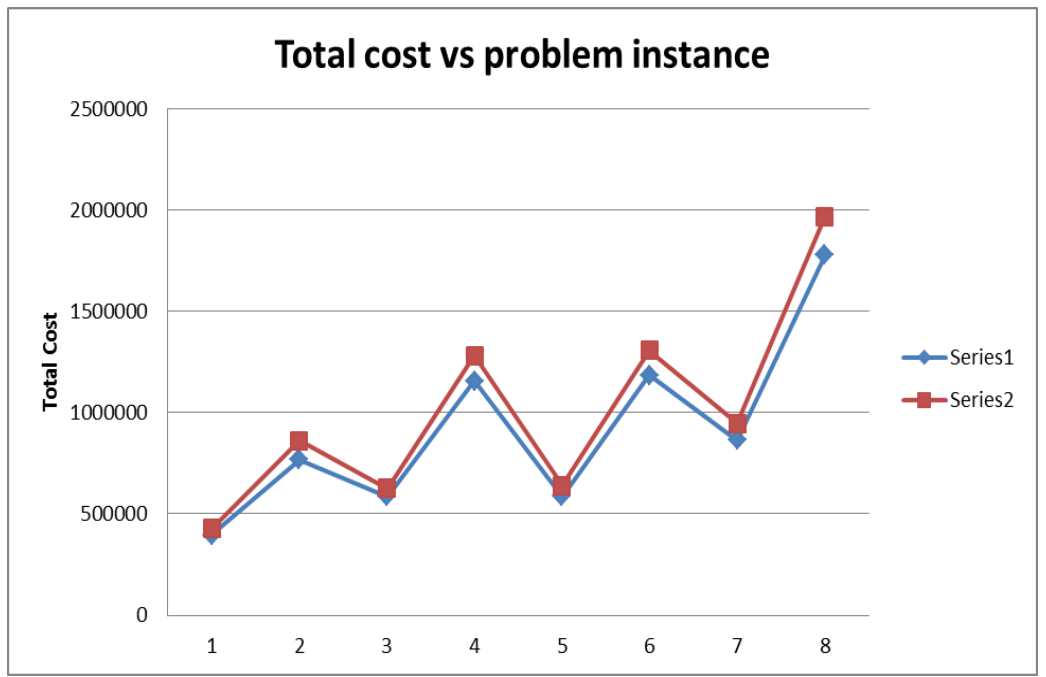

Figure 3. Comparison of GA \& CPLEX solution total costs series $1=$ CPLEX data, series $2=$ GA data.

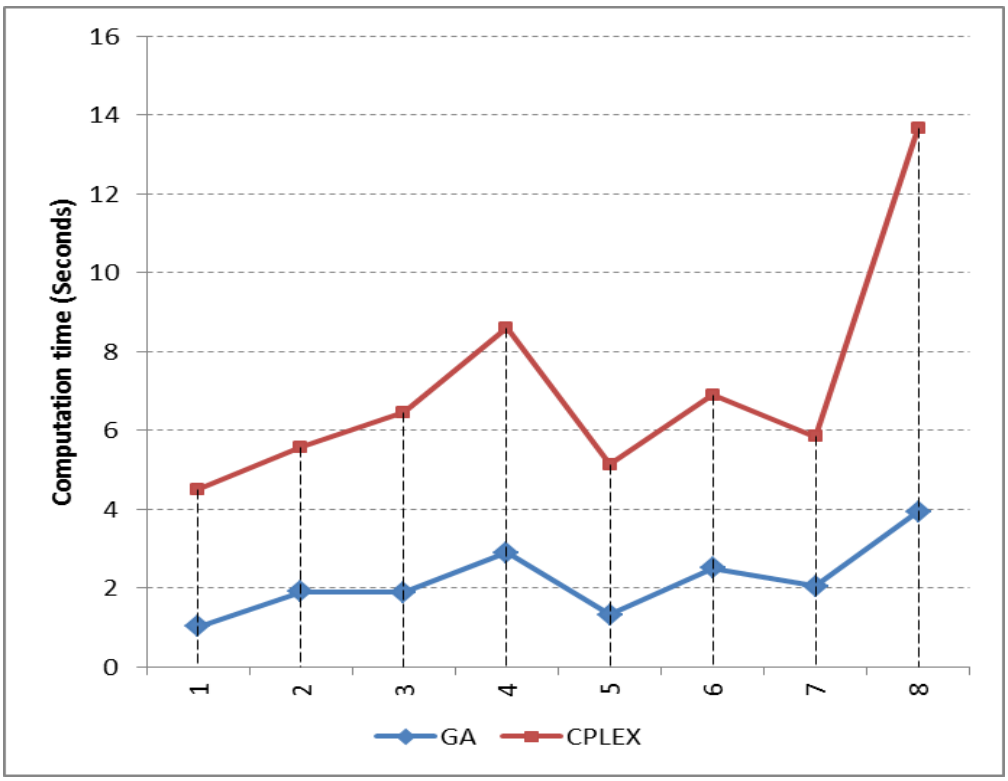

Figure 4. Comparison of GA CPLEX solution computation time. 
International Journal of Mathematical, Engineering and Management Sciences

Vol. 6, No. 3, 961-974, 2021

https://doi.org/10.33889/IJMEMS.2021.6.3.057

\section{Conclusions and Future Research}

While a few prior studies have proposed various solution approaches to solve MPCLSP-IT problems, the prior methods were limited in scope, in problem size. This paper proposes the genetic algorithm approach to solve a large scale MPCLSP-IT problem. The feasibility of the solutions obtained from the GA and CPLEX was verified. It can be observed from competitive analysis that GA could produce a near optimal solution with a reasonable less than $14 \%$ solution gap in a substantially smaller amount of computation time (i.e. on average less than 1 second). That being said, this study can be extended in the future in multiple ways. First, future efforts will include the development of more efficient heuristics to narrow the solution gap. Next, the current model does not consider the back-ordering and shortage costs which are also prevalent in realworld applications. Therefore, the consideration of these aspects will strengthen the efficacy and utility of the model (Badhotiya et al., 2018; Badhotiya et al., 2019). Future work can also extend the number of echelons in a supply chain beyond the two-echelon problem as was the case in this study. Apart from that, novel and computationally efficient metaheuristics proposed in the literature, such as Grey Wolf Optimization (Negi et al., 2021), Flower Pollination Algorithm (Pant et al., 2017a), Cuckoos Search Algorithm, have demonstrated promising results in other research areas (Kumar et al., 2019; Pant et al., 2017b; Uniyal et al., 2020). This study can be extended by exploring one of these metaheuristics to obtain better results.

\section{Conflict of Interest}

The authors confirm that there is no conflict of interest to declare for this publication.

\section{Acknowledgements}

This research did not receive any specific grant from funding agencies in the public, commercial, or not-for-profit sectors. The authors would like to thank the editor and anonymous reviewers for their comments that help improve the quality of this work.

\section{References}

Almada-Lobo, B., Klabjan, D., Antónia Carravilla, M.A., \& Oliveira, J.F. (2007). Single machine multiproduct capacitated lot sizing with sequence-dependent setups. International Journal of Production Research, 45(20), 4873-4894. https://doi.org/10.1080/00207540601094465.

Almeder, C. (2010). A hybrid optimization approach for multi-level capacitated lot-sizing problems. European Journal of Operational Research, 200(2), 599-606. https://doi.org/10.1016/j.ejor.2009.01.019.

Aytug, H., Khouja, M., \& Vergara, F.E. (2003). Use of genetic algorithms to solve production and operations management problems: a review. International Journal of Production Research, 41(17), 3955-4009. https://doi.org/10.1080/00207540310001626319.

Baccouche, M., Boukachour, J., \& Benabdelhafid, A. (2005). Hybrid genetic algorithm for the generalised assignment problem. Journal of Decision Systems, 14(1-2), 83-107. https://doi.org/10.3166/jds.14.83107

Badhotiya, G.K., Soni, G., \& Mittal, M.L. (2018). An analysis of mathematical models for multi-site production and distribution planning. International Journal of Intelligent Enterprise, 5(4), 309-332. https://doi.org/10.1504/IJIE.2018.095714. 
International Journal of Mathematical, Engineering and Management Sciences

Vol. 6, No. 3, 961-974, 2021

https://doi.org/10.33889/IJMEMS.2021.6.3.057

Badhotiya, G.K., Soni, G., \& Mittal, M.L. (2019). Fuzzy multi-objective optimization for multi-site integrated production and distribution planning in two echelon supply chain. The International Journal of Advanced Manufacturing Technology, 102(1), 635-645. https://doi.org/10.1007/s00170-018-3204-2.

Brahimi, N., Absi, N., Dauzère-Pérès, S., \& Nordli, A. (2017). Single-item dynamic lot-sizing problems: an updated survey. European Journal of Operational Research, 263(3), 838-863.

Brandimarte, P. (2006). Multi-item capacitated lot-sizing with demand uncertainty. International Journal of Production Research, 44(15), 2997-3022. https://doi.org/10.1080/00207540500435116.

Buschkühl, L., Sahling, F., Helber, S., \& Tempelmeier, H. (2010). Dynamic capacitated lot-sizing problems: a classification and review of solution approaches. Or Spectrum, 32(2), 231-261. https://doi.org/10.1007/s00291-008-0150-7.

Carvalho, D.M., \& Nascimento, M.C.V. (2016). Lagrangian heuristics for the capacitated multi-plant lot sizing problem with multiple periods and items. Computers \& Operations Research, 71, 137-148. https://doi.org/10.1016/j.cor.2016.01.019.

Carvalho, D.M., \& Nascimento, M.C.V. (2018). A kernel search to the multi-plant capacitated lot sizing problem with setup carry-over. Computers \& Operations Research, 100, 43-53. https://doi.org/10.1016/j.cor.2018.07.008.

de Armas, J., \& Laguna, M. (2019). Parallel machine, capacitated lot-sizing and scheduling for the pipeinsulation industry. International Journal of Production Research, 58(3), 800-817. https://doi.org/10.1080/00207543.2019.1600763.

Deleplanque, S., Kedad-Sidhoum, S., \& Quilliot, A. (2013). Lagrangean heuristic for a multi-plant lotsizing problem with transfer and storage capacities. RAIRO-Operations Research, 47(4), 429-443. https://doi.org/10.1051/ro/2013050.

Deleplanque, S., Quilliot, A., \& Kedad-Sidhoum, S. (2012). Decomposing a multi-plant, multi-item lotsizing problem with transfer costs/capacities into facility location and flow sub-problems. IFAC Proceedings Volumes, 45(6), 1433-1438. https://doi.org/10.3182/20120523-3-RO-2023.00062.

Ghirardi, M., \& Amerio, A. (2019). Matheuristics for the lot sizing problem with back-ordering, setup carry-overs, and non-identical machines. Computers \& Industrial Engineering, 127, 822-831. https://doi.org/10.1016/j.cie.2018.11.023.

Goldberg, D.E., \& Holland, J.H. (1988). Genetic algorithms and machine learning. Machine Learning, 3(2), 95-99. https://doi.org/10.1023/A:1022602019183.

González-Neira, E.M., \& Montoya-Torres, J.R. (2017). A GRASP meta-heuristic for the hybrid flowshop scheduling problem. Journal of Decision Systems, 26(3), https://doi.org/10.1080/12460125.2017.1351863.

Gopalakrishnan, M., Ding, K., Bourjolly, J.M., \& Mohan, S. (2001). A tabu-search heuristic for the capacitated lot-sizing problem with set-up carryover.Management Science,47(6), 851-863. https://doi.org/10.1287/mnsc.47.6.851.9813.

Goren, H.G., Tunali, S., \& Jans, R. (2012). A hybrid approach for the capacitated lot sizing problem with setup carryover. International Journal of Production Research, 50(6), 1582-1597. https://doi.org/10.1080/00207543.2011.559486.

Gruson, M., Bazrafshan, M., Cordeau, J.F., \& Jans, R. (2019). A comparison of formulations for a threelevel lot sizing and replenishment problem with a distribution structure. Computers \& Operations Research, 111, 297-310. https://doi.org/10.1016/j.cor.2019.07.005.

Guinet, A. (2001). Multi-site planning: a transshipment problem. International Journal of Production Economics, 74(1-3), 21-32. https://doi.org/10.1016/S0925-5273(01)00104-9. 
International Journal of Mathematical, Engineering and Management Sciences

Vol. 6, No. 3, 961-974, 2021

https://doi.org/10.33889/IJMEMS.2021.6.3.057

Goren, H.G., Tunali, S., \& Jans, R. (2010). A review of applications of genetic algorithms in lot sizing. Journal of Intelligent Manufacturing, 21(4), 575-590. https://doi.org/10.1007/s10845-0080205-2.

Gupta, D., \& Magnusson, T. (2005). The capacitated lot-sizing and scheduling problem with sequencedependent setup costs and setup times. Computers \& Operations Research, 32(4), 727-747. https://doi.org/10.1016/j.cor.2003.08.014.

Hetreux, G., Merce, C., \& Fontan, G. (1996). Multi-stage, multi-product planning: a hierarchical approach using time aggregation. Journal of Decision Systems, 5(1-2), 35-50. https://doi.org/10.1080/12460125.1996.10511673.

Hwang, H.C., \& Kang, J. (2020). The two-level lot-sizing problem with outbound shipment. Omega, 90, 101997. https://doi.org/10.1016/j.omega.2018.11.006.

Jans, R., \& Degraeve, Z. (2007). Meta-heuristics for dynamic lot sizing: a review and comparison of solution approaches. European Journal of Operational Research,177(3), 1855-1875. https://doi.org/10.1016/j.ejor.2005.12.008.

Kelly Jr, J.D., \& Davis, L. (1991, August). A hybrid genetic algorithm for classification. In International Joint Conferences on Artificial Intelligence (Vol. 91, pp. 645-650).

Karimi, B., Ghomi, S.F., \& Wilson, J.M. (2003). The capacitated lot sizing problem: a review of models and algorithms. Omega, 31(5), 365-378. https://doi.org/10.1016/S0305-0483(03)00059-8.

Kumar, A., Pant, S., \& Ram, M. (2019). Gray wolf optimizer approach to the reliability-cost optimization of residual heat removal system of a nuclear power plant safety system. Quality and Reliability Engineering International, 35(7), 2228-2239. https://doi.org/10.1002/qre.2499.

Maes, J., McClain, J.O., \& Van Wassenhove, L.N. (1991). Multilevel capacitated lotsizing complexity and LP-based heuristics. European Journal of Operational Research,53(2), 131-148. https://doi.org/10.1016/0377-2217(91)90130-N.

Nascimento, M.C.V., Resende, M.G.C., \& Toledo, F.M. (2010). GRASP heuristic with path-relinking for the multi-plant capacitated lot sizing problem. European Journal of Operational Research, 200(3), 747-754. https://doi.org/10.1016/j.ejor.2009.01.047.

Nascimento, M.C.V., \& Toledo, F.M.B. (2008). A hybrid heuristic for the multi-plant capacitated lot sizing problem with setup carry-over. Journal of the Brazilian Computer Society, 14(4), 7-15. https://doi.org/10.1007/BF03192568.

Negi, G., Kumar, A., Pant, S., \& Ram, M. (2021). GWO: a review and applications. International Journal of System Assurance Engineering and Management, 12(1),1-8. https://doi.org/10.1007/s13198-02000995-8.

Mohammadi, M., Musa, S.N., \& Omar, M.B. (2020). Optimisation of multi-plant capacitated lot-sizing problems in an integrated supply chain network using calibrated metaheuristic algorithms. International Journal of Operational Research,39(3), 325-363. https://doi.org/10.1504/ijor.2020.10029302.

Özdamar, L., \& Barbarosoglu, G. (2000). An integrated Lagrangean relaxation-simulated annealing approach to the multi-level multi-item capacitated lot sizing problem. International Journal of Production Economics, 68(3), 319-331. https://doi.org/10.1016/S0925-5273(99)00100-0.

Pant, S., Kumar, A., \& Ram, M. (2017a). Flower pollination algorithm development: a state of art review. International Journal of System Assurance Engineering and Management, 8(2), 1858-1866. https://doi.org/10.1007/s13198-017-0623-7.

Pant, S., Kumar, A., Bhan, S., \& Ram, M. (2017b). A modified particle swarm optimization algorithm for nonlinear optimization. Nonlinear Studies, 24(1), 127-135. 
International Journal of Mathematical, Engineering and Management Sciences

Vol. 6, No. 3, 961-974, 2021

https://doi.org/10.33889/IJMEMS.2021.6.3.057

Quadt, D., \& Kuhn, H. (2008). Capacitated lot-sizing with extensions: a review. 4OR, 6(1), 61-83. https://doi.org/10.1007/s10288-007-0057-1.

Sahling, F., Buschkühl, L., Tempelmeier, H., \& Helber, S. (2009). Solving a multi-level capacitated lot sizing problem with multi-period setup carry-over via a fix-and-optimize heuristic. Computers \& Operations Research, 36(9), 2546-2553. https://doi.org/10.1016/j.cor.2008.10.009.

Sambasivan, M., \& Schmidt, C.P. (2002). A heuristic procedure for solving multi-plant, multi-item, multiperiod capacitated lot-sizing problems. Asia Pacific Journal of Operational Research, 19(1), 87-106.

Sambasivan, M., \& Yahya, S. (2005). A Lagrangean-based heuristic for multi-plant, multi-item, multiperiod capacitated lot-sizing problems with inter-plant transfers. Computers \& Operations Research, 32(3), 537-555. https://doi.org/10.1016/j.cor.2003.08.002.

Uniyal, N., Pant, S., \& Kumar, A. (2020). An overview of few nature inspired optimization techniques and its reliability applications. International Journal of Mathematical, Engineering and Management Sciences, 5(4), 732-743. https://doi.org/10.33889/IJMEMS.2020.5.4.058.

Vercellis, C. (1999). Multi-plant production planning in capacitated self-configuring two-stage serial systems. European Journal of Operational Research, 119(2), 451-460. https://doi.org/10.1016/S03772217(99)00146-0.

Vincent, B., Duhamel, C., Ren, L., \& Tchernev, N. (2020). A population-based metaheuristic for the capacitated lot-sizing problem with unrelated parallel machines. International Journal of Production Research, 58(21), 6689-6706. https://doi.org/10.1080/00207543.2019.1685699. 